\title{
USRP Testbed and Performance Analysis of New Reconfigurable LDACS In Presence of DME Interference
}

\author{
Niharika Agrawal, Himani Joshi and S. J. Darak \\ Algorithms to Architectures Lab, \\ ECE, IIIT-Delhi, India-110020 \\ Email:\{niharikaa, himanij, sumit\}@iiitd.ac.in
}

\author{
Faouzi Bader \\ SCEE/IETR Lab \\ CentraleSuplec campus of Rennes, France \\ Email: faouzi.bader@supelec.fr
}

\begin{abstract}
Existing VHF band based air-to-ground communication (A2GC) is suffering from severe congestion especially in Europe and the US where air-traffic has increased significantly in the last decade. To overcome this problem, a new $L$-band digital aeronautical communication system (LDACS) is being explored by ICAO. Recently, we proposed reconfigurable filtered OFDM based LDACS (Ref-OFDM) which has the potential to improve the spectrum efficiency of existing OFDM based LDACS. In this paper, we develop an experimental setup using universal software radio peripherals (USRPs) and analyze the performance of these two LDACS. The comparison is made in terms of interference to the legacy distance measuring equipment (DME) channels in $L$-band and BER for various channel conditions. Specifically, we analyze the performance by varying three parameters related to the LDACS/DME transmitter and receiver: 1) Altitude, 2) Distance, and 3) DME antenna gain. We also consider line-ofsight (LOS) as well as non-LOS paths. The proposed Ref-OFDM performs superior in all considered scenarios, and experimental results closely match the simulation results making Ref-OFDM based LDACS as an attractive alternative to the next generation LDACS.
\end{abstract}

Keywords-Air traffic management (ATM), Reconfigurable filtered OFDM (Ref-OFDM), Air to ground communication (A2GC), L-band Digital Aeronautical Communication System (L-DACS)

\section{INTRODUCTION}

The Air traffic management (ATM) system handles all the communication between the ground terminals, aircrafts in airspace as well as between the aircrafts and ground terminals. Over the years, the air conveyance has increased immensely, and the current aeronautical communication system is reaching its capacity limit in high-density areas like Europe and the USA. In 2002, the International Civil Aviation Organization (ICAO) realized the need to improve the existing ATM system. The modernization of the ATM system is currently pursued by the Next Generation Air Transportation System (NextGen) and Single European Sky ATM Research (SESAR) in the USA and Europe respectively. They are working on the Future Communication Infrastructure (FCI) system to meet the exponentially increasing air-traffic and support a wide variety of services and applications [1-3]. It consists of several communication data links between satellite stations, aircraft and ground terminals. Such FCI system is also used for other communications, navigation, and surveillance (CNS) applications. In this work, we mainly focus on the air-to-ground communication (A2GC) link which is the most crucial data link within the FCI, and it is shown in Fig. 1.

The A2GC has evolved tremendously in recent years. The first voice-based air to ground communication was deployed in the 1940s, used analog modulation scheme. To further increase

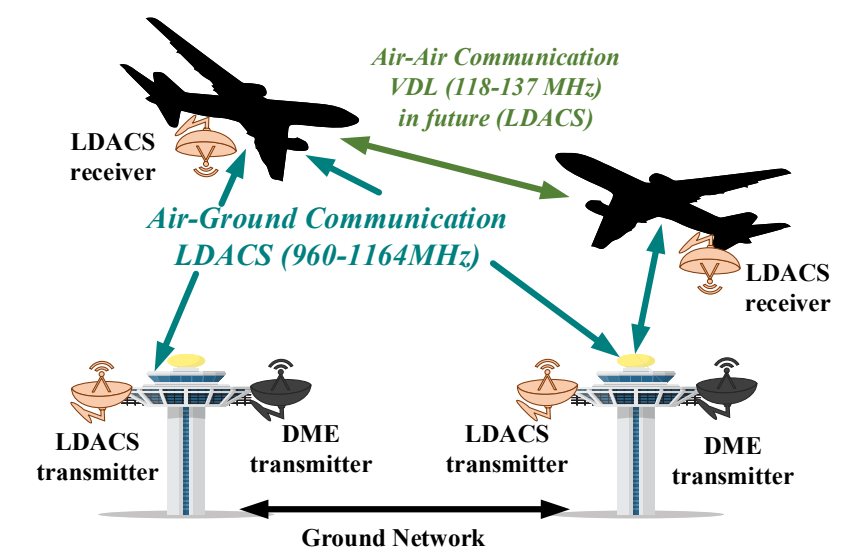

Fig. 1. Air-to-Ground communication data link of the LDACS with DME interference.

the throughput and robustness of the system, the digitization of A2GC system was done in 1990s [2]. This digital A2GC link was deployed in the VHF band (118-137 MHz) and hence, referred to as VHF Data Link (VDL). It is currently used in the A2GC and air-to-air communications [2-5]. In the last decade, the air traffic has increased significantly leading to severe congestion in the VHF band. Furthermore, the existing system cannot support high-speed multimedia services due to limited bandwidth [6].

To meet the emergent demand of communication capacity, $L$-band (960-1164 MHz) has been allocated for the A2GC link in 2007 and referred to as $L$-band digital aeronautical communication system (LDACS). The LDACS is expected to be deployed in multiple $1 \mathrm{MHz}$ vacant frequency bands between adjacent legacy distance measuring equipment (DME) channels [7]. The first version of the LDACS is based on the orthogonal frequency-division multiplexing (OFDM) and frequency-division duplex (FDD). Due to the rectangular filtering in the time domain, OFDM has very high side lobe attenuation; hence, high interference with the adjacent legacy user leads to the limited transmission of the bandwidth of $498 \mathrm{KHz}$ and therefore, less than $50 \%$ spectrum utilization. This opens the opportunity to explore the other candidate waveforms as an alternative to the OFDM based LDACS. Recently, we proposed reconfigurable filtered OFDM based LDACS (RefOFDM) which can adapt the transmission bandwidths on the fly and uses a single variable digital filter for all the different transmission bandwidths [8,9]. This enables wider transmission along with the multiple narrowband communications to fulfill the spectrum requirement for futuristic services and 
support multimedia data. Via theoretical and simulation results, we show that proposed LDACS offers superior performance in terms of power spectral density (PSD) and bit-error-rate (BER) without compromising significantly on the complexity $[8,9]$. This paper aims to support our claims via experimental results in the real radio environment. The main contributions of the paper are:

1) We develop an experimental testbed using three $\mathrm{Na}$ tional Instrument (NI) USRP-2922 and VERT900 antennas for the deployment of the proposed Ref-OFDM based LDACS between two DME channels. Among three, one USRP is used for DME transmission as well as reception while the other two USRPs are used as LDACS transmitter and receiver, respectively.

2) The performance of the proposed Ref-OFDM based LDACS is compared with the existing LDACS in terms of interference (PSD) to the legacy DME channels, bit error rate, and the throughput achieved. For illustration, we consider two transmission bandwidths: (1) $498 \mathrm{KHz}$ and (2) $732 \mathrm{KHz}$.

3) We analyze the performance by varying the parameters related to LDACS/DME transmitter and a receiver like 1) Distance, 2) Altitude, and 3) DME antenna gain.

The rest of the paper is structured as follows. Section II gives a brief overview of the proposed Ref-OFDM based LDACS transceiver. The developed USRP testbed for LDACS deployment is presented in section III followed by the experimental results in section IV. Section V concludes the paper.

\section{REF-OFDM BASED LDACS TRANSCEIVER}

With the growing demand for air traffic, flight safety and good signal transmission is a major goal for the future A2GC. To fulfill the spectrum requirements, we have recently proposed an alternative to OFDM based LDACS referred to Ref-OFDM based LDACS protocol in [9]. In this section, a brief overview is provided for better understanding of the proposed testbed.

Due to the very high out of band emission, the existing OFDM based LDACS uses $498 \mathrm{KHz}$ transmission bandwidth out of $1 \mathrm{MHz}$ spectral gap between two adjacent DME channels which leads to more than $50 \%$ of spectrum wastage. The proposed Ref-OFDM based LDACS uses a bandwidth reconfigurable fixed coefficient multi-band linear phase FIR filter in the time domain, in which a single prototype filter can accommodate different transmission bandwidths. The proposed Ref-OFDM based LDACS has very less power in side lobes; hence, the interference with the adjacent DME channels is also less; this leads to the increase in the transmission bandwidth.
Also, because of the side lobe filtering, there is no need for guard bands. Therefore, it provides higher throughput compared to the existing one. The Ref-OFDM enables a transmission in multiple narrowband and allows to switch the transmission bandwidths on the fly.

A new frame structure protocol for Ref-OFDM based LDACS using the standard 128 point FFT size and $9.76 \mathrm{KHz}$ subcarrier spacing is proposed in [9], which can transmit in 186- $732 \mathrm{KHz}$ bandwidth range, The detailed description of the frame structure for the proposed Ref-OFDM is discussed in [9]. The bandwidth reconfigurable filter is designed using Coefficient Decimation factor (CDM) and improved CDM (ICDM) method. In this method, a baseband bandpass prototype filter $F\left(e^{j \omega_{c}}\right)$ is designed with the cut-off frequency $\omega_{c}$. The filter coefficients are obtained using the Parks-McClellan optimal FIR filter design method. The CDM generates a low pass filter response having the cut-off frequency as $D \omega_{c}$ where $D \in\{1,2,3 .$.$\} by keeping only the D^{t h}$ coefficients unchanged and discarded the remaining coefficients. Similarly, the ICDM filter generates high pass filter response of cut-off frequency $1-D \omega_{c}$, from which low pass response can be easily obtained using the appropriate delayed version of the input signal. In the ICDM method, every $D^{t h}$ coefficient are kept with a sign reversal of every other coefficient, and the remaining coefficients are discarded.

The block diagram of the proposed Ref-OFDM based LDACS is shown in Fig. 2. As per the LDACS specifications [10], the LDACS randomizer first randomizes the input bitstream according to the randomizer sequence and then the randomized data is encoded by convolutional or Reed Solomon encoder. The output bits are then converted to symbols using modulator by selecting the appropriate modulation scheme such as QPSK, 16 QAM, 64 QAM. These symbols are then mapped to the subcarriers according to the LDACS frame structure followed by the conventional IFFT and cyclic prefix (CP) addition. The output of the CP addition is filtered using the proposed bandwidth reconfigurable digital filter; the filtered data is appropriately up sampled and converted to the RF center frequency and transmitted over the air. The signal is then down-converted to the same localized baseband frequency and filtered using the same filter at the receiver. Similar and reverse operations are performed at the receiver.

\section{USRP TESTBED FOR LDACS}

In this section, we present experimental testbed using Universal Software Radio Peripheral (USRP) for the deployment of the LDACS. USRP is a tunable transceiver from NI and is widely used for prototyping wireless communication systems.

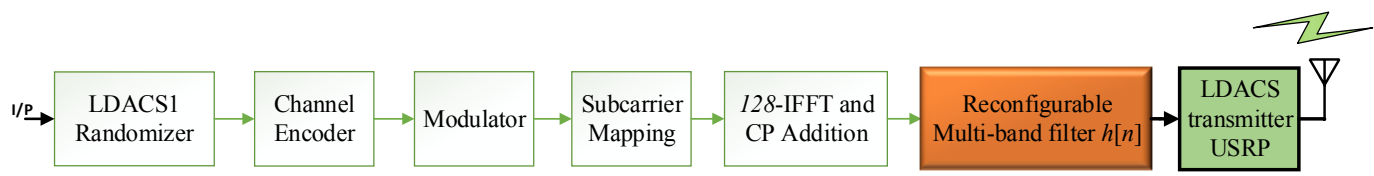

(a)
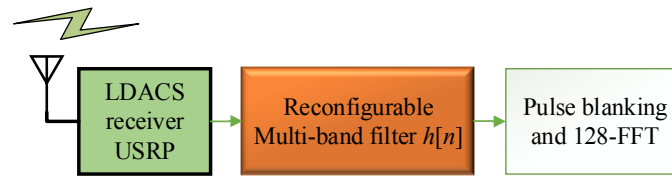

Channel Estimation

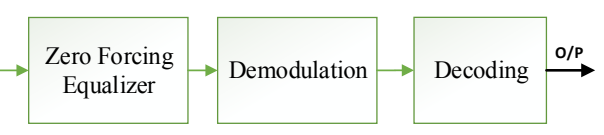

(b)

Fig. 2. Block diagram of the proposed Ref-OFDM based LDACS transceiver. 
TABLE I. PARAMETERS OF TRANSMITTER AND RECEIVER USRP

\begin{tabular}{|l|l|l|}
\hline Parameters & Transmitter & Receiver \\
\hline Carrier Frequency & $985 \mathrm{MHz}$ & $985 \mathrm{MHz}$ \\
\hline IQ Sampling Rate & $1.1 \mathrm{MHz}$ & $1.1 \mathrm{MHz}$ \\
\hline LDACS antenna gain & $2 \mathrm{~dB}$ & $2 \mathrm{~dB}$ \\
\hline DME antenna gain & $2 \mathrm{~dB} / 10 \mathrm{~dB}$ & - \\
\hline Acquisition Duration & $\mathrm{NA}$ & $1 \mathrm{~s}$ \\
\hline
\end{tabular}

The Proposed testbed consists of three National Instrument (NI) USRP-2922 and VERT900 antennas as shown in Fig. 3. One USRP is used for DME transmission as well as reception while other two USRPs are used as LDACS transmitter and receiver, respectively. Several baseband signal processing blocks of the transceiver are realized using the LabView environment from NI.

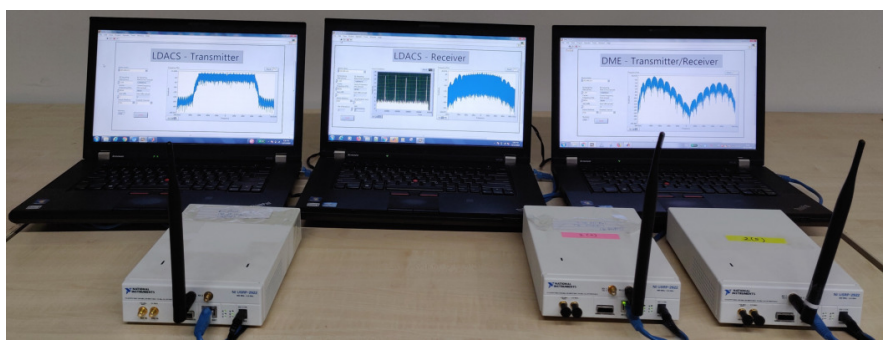

Fig. 3. USRP based testbed for LDACS.

\section{A. LDACS Transmitter}

To implement the inlay approach of deployment, the proposed Ref-OFDM based LDACS signal is transmitted at the carrier frequency of $985 \mathrm{MHz}$ with the IQ sampling rate of 1.1 MHz. The transmission has been performed for the variable bandwidths ranging from $186-732 \mathrm{KHz}$ using 128 points IFFT with $9.76 \mathrm{KHz}$ sub-carrier spacing.

The LDACS transmitter realization is shown in Fig. 4, consists of three subsystems: (a) the first subsystem sets the transmission parameters such as carrier frequency, antenna gain, IQ sampling rate, and transmission antenna port, (b) the second subsystem is the LDACS transmitter which first generates the random bits and then modulates them by selecting an appropriate modulation scheme. The "OFDM Block.vi " performs all the LDACS transmitter operations as discussed in section II. The synchronization symbols are added to the LDACS frame for the synchronization at the receiver followed by the filtering operation performed using the "Reconfigurable Multiband Filter.vi ", (c) The Third subsystem continuously transmits the filtered data over the desired carrier frequency via LDACS USRP transmitter.

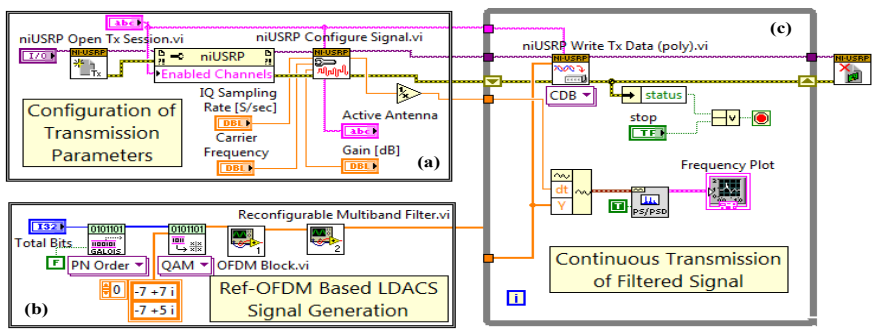

Fig. 4. Implementation of Ref-OFDM based LDACS transmitter in the LabView environment

\section{B. DME transmitter}

The DME is a navigation legacy system composed of Gaussian shaped pulse pairs having $1 \mathrm{MHz}$ of spectral gap and variance of $4.5 * 10^{11}$. More details of the DME specifications and its time and frequency domain representation is provided in [8].

Similar to the LDACS transmitter, the DME transmitter model as shown in Fig 5 comprises three sub-blocks (a) the first sub-block configures the USRP parameters as mentioned in the table I for the DME transmission, (b) the second sub-block is dedicated for the DME signal generation. This includes two Gaussian monopulse generator blocks which generate the Gaussian pulses with the center frequencies of 984.5 $\mathrm{MHz}$ and $985.5 \mathrm{MHz}$. These monopulses are later combined to achieve the DME signal of Gaussian pulse pair, and at every transmission, it transmits 2700 pulse pairs per second, (c) the last sub-block is realized to continuously transmit the DME signal over the air.

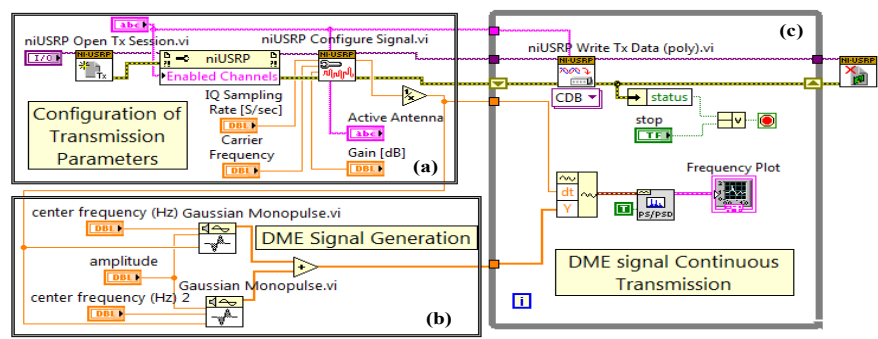

Fig. 5. Implementation of DME transmitter in the LabView environment

\section{LDACS Receiver}

The LDACS signal is then received by the LDACS receiver USRP, tuned at $985 \mathrm{MHz}$ to receive the transmitted signal at IQ sampling rate of $1.1 \mathrm{MHz}$. However, due to a mismatch between the oscillator frequency at the transmitter and receiver USRPs, additional synchronization is needed at the receiver (see details in Fig. 6). In this work, to identify the beginning of each frame, the received signal is frame synchronized by determining the cross-correlation between the received signal and the known synchronization symbols added in the LDACS frame at the transmitter. The signal is then processed from the maximum correlated value index. The proposed Ref-OFDM uses a matched reconfigurable filter at the receiver followed by fine synchronization to estimate time and frequency offsets. The fine synchronization is based on the correlation of cyclic prefix of each OFDM symbol. After that, the channel is estimated using pilots followed by channel equalization via zero forcing method. In the end, the symbols are demodulated and decoded for subsequent performance analysis.

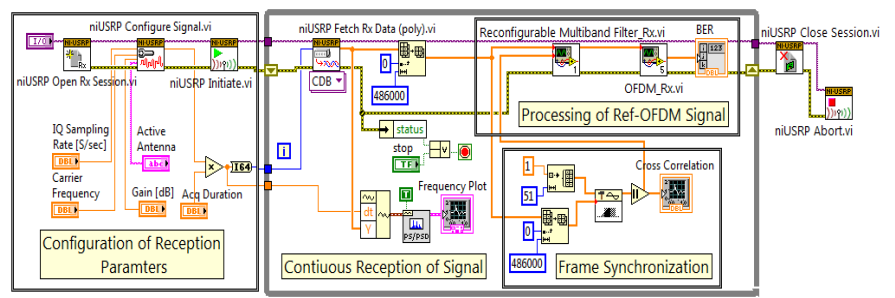

Fig. 6. Implementation of Ref-OFDM based LDACS receiver in the LabView environment 
In addition to proposed LDACS, we have also realized existing OFDM based LDACS along with additional windowing. We have skipped the details due to limited space constraints.

\section{HARDWARE EXPERIMENTAL RESULTS}

For experimental results and analysis, the parameters are considered as per LDACS specifications and are given in table II. We have done the comparison in terms of the PSD, BER and throughput for two different transmission bandwidths: 1) $498 \mathrm{KHz}$ and 2) $732 \mathrm{KHz}$.

\section{TABLE II. LDACS SPECIFICATIONS}

\begin{tabular}{|c|c|}
\hline Parameters & Value \\
\hline Total Bandwidth & $1.1 \mathrm{MHz}$ \\
Transmitted Bandwidth & any of the supported bandwidth $(186-732 \mathrm{KHz})$ \\
Length of FFT & 128 \\
Sub-carrier spacing & $9.76 \mathrm{KHz}$ \\
Used sub-carriers & $18-74$ \\
Total Symbol duration & $120 \mu \mathrm{s}$ \\
Modulation Scheme & QPSK \\
CC encoder rate & 0.5 \\
RS encoder rate & 0.9 \\
\hline
\end{tabular}

\section{A. Power Spectral Density Analysis}

The PSD performance comparison of the existing LDACS and the proposed LDACS is shown in Fig. 7 (a) and (b) for the considered $498 \mathrm{KHz}$ and $732 \mathrm{KHz}$ transmission bandwidth respectively. The existing LDACS can only transmit up to $498 \mathrm{KHz}$ due to the very high side lobe power creating the interference higher than the $40 \mathrm{~dB}$ tolerable limit of the DME signal. It can be observed from the Fig. 7 (a) and (b) that the proposed LDACS has approximately $35 \mathrm{~dB}$ less out of band (OOB) emission due to the reconfigurable filtering, hence, the less adjacent DME channel interference. This allows the proposed LDACS to achieve higher transmission bandwidth up to $732 \mathrm{kHz}$, which leads to relatively $25 \%$ of the spectral improvement than the existing one.

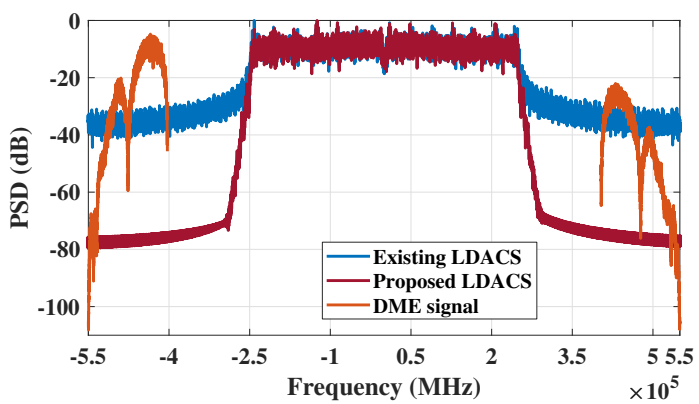

(a)

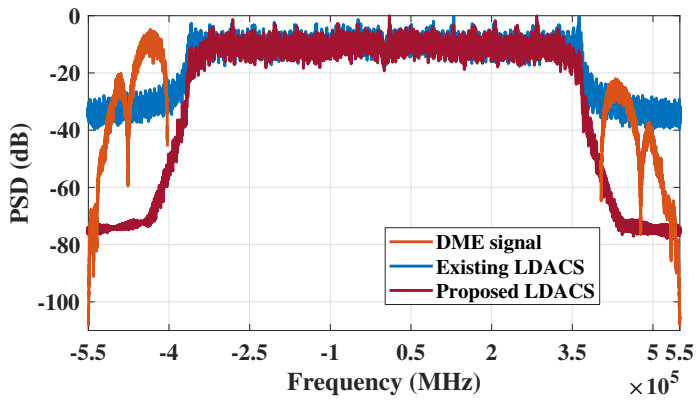

(b)

Fig. 7. The PSD comparison for two different transmission bandwidths, (a) $498 \mathrm{KHz}$, and (b) $732 \mathrm{KHz}$

\section{B. Bit error Rate Analysis}

Here, we compare the BER performance of the proposed LDACS with the existing LDACS in the presence of the DME interference. The LDACS signal received at the receiver USRP is presented in the Fig 8 for two different transmission bandwidths (1) $498 \mathrm{KHz}$, and (2) $732 \mathrm{KHz}$. As we can observe that $732 \mathrm{KHz}$ bandwidth accommodates very high DME interference, which consequently results in lower BER performance.

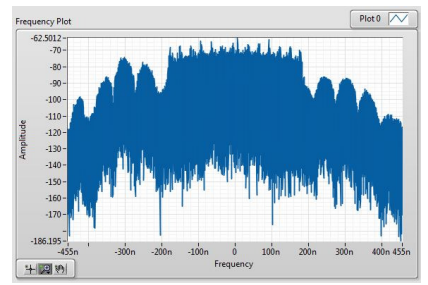

(a)

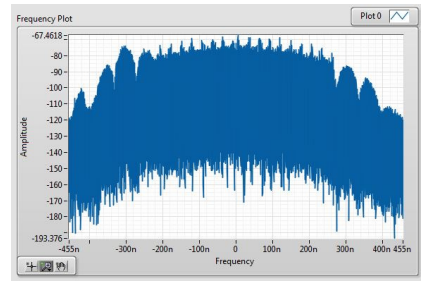

(b)
Fig. 8. Signal received at LDACS receiver USRP: (a) $498 \mathrm{KHz}$, (b) 732 KHz.

Next, we discuss the detailed BER analysis by considering three scenarios (1) variable distance, (2) variable altitude, and (3) variable DME antenna gain.

1) Variable Distance: Here, the BER performance is analyzed by varying the distance $(D)$ between the LDACS/DME transmitter and the LDACS receiver considering $498 \mathrm{KHz}$ and $732 \mathrm{KHz}$ as the transmission bandwidths. We assume that DME and LDACS transmitter are located close to each

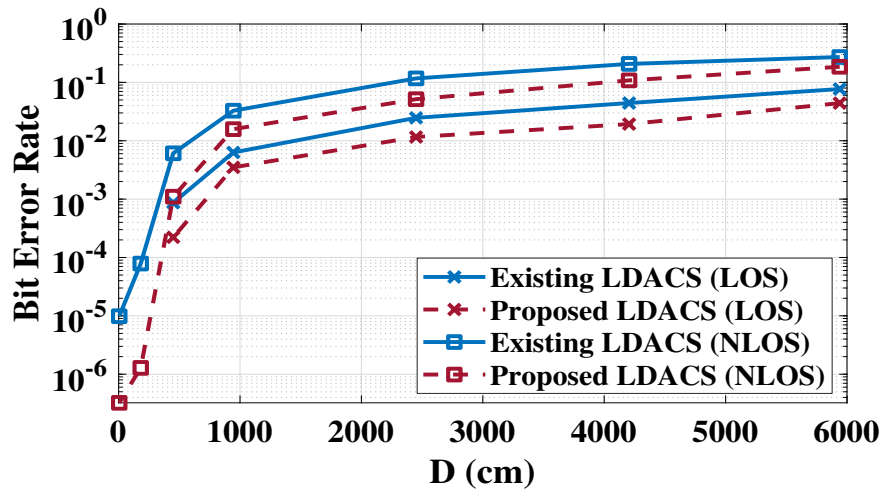

(a)

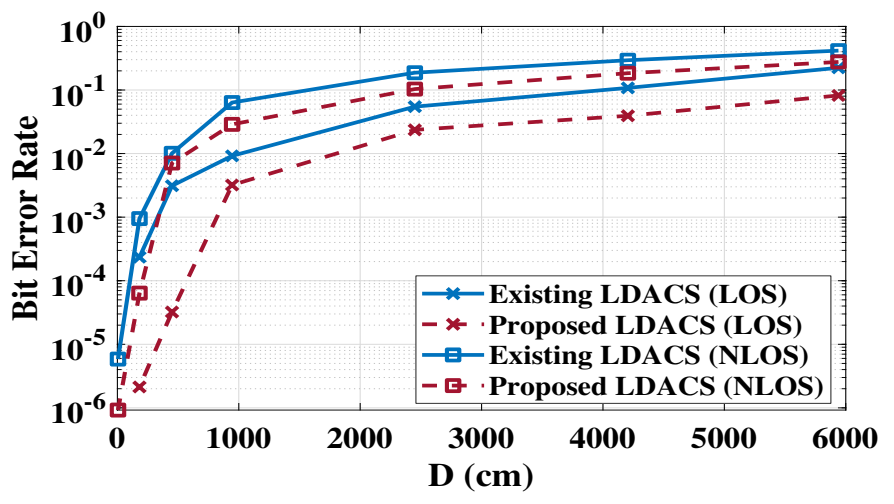

(b)

Fig. 9. The BER comparison for the variable distance between LDACS/DME transmitter and LDACS receiver $(D)$ for two different transmission bandwidths, (a) $498 \mathrm{KHz}$, and (b) $732 \mathrm{KHz}$ 
other resulting in worst possible interference. For all the distances, line of sight (LOS) and non-line of sight (NLOS) communications are examined. As shown in Fig. 9, the BER degraded with an increase in $D$ and proposed LDACS offers better performance than existing LDACS. It can be observed that BER gets worse for NLOS scenario as well as for higher transmission bandwidth.

2) Variable Altitude and DME antenna gain: Next, we analyze the BER performance by varying the altitude of the LDACS receiver keeping the LDACS/DME transmitter at the same fixed level. For all the heights, two values of the DME antenna gain (1) $2 \mathrm{~dB}$ and (2) $10 \mathrm{~dB}$ are considered. When the LDACS receiver is placed at a higher altitude, the received LDACS signal power reduces while the interference power remains the same. Thus the BER performance degrades as shown in Fig. 10. At the same time, with the increase in DME antenna gain (from $2 \mathrm{~dB}$ to $10 \mathrm{~dB}$ ) the DME interference power dominates the LDACS transmitted signal power which causes in higher BER.

The proposed LDACS has better BER performance than the existing one for all the considered scenarios makes it a very enticing replacement to the existing W-OFDM based LDACS.

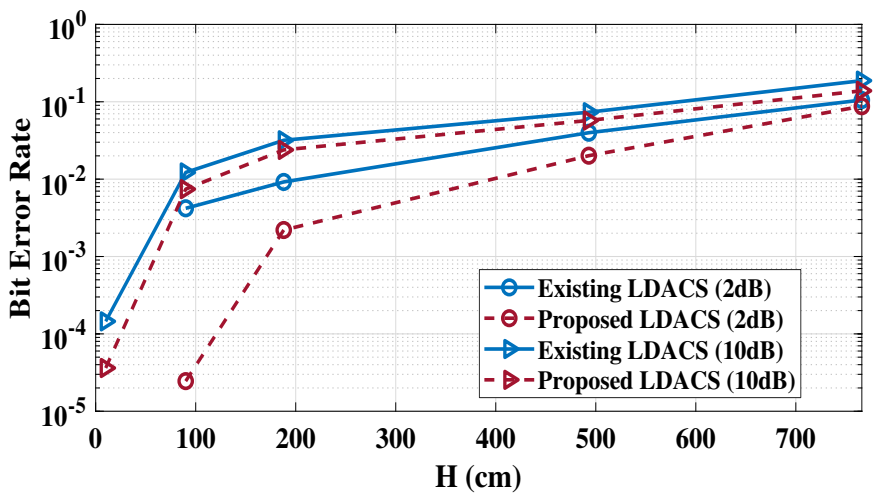

(a)

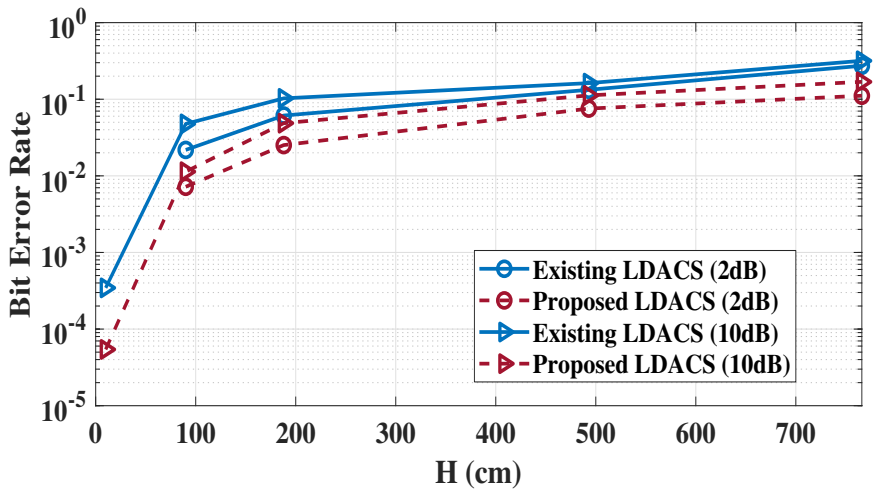

(b)

Fig. 10. The BER comparison for the variable altitude of the LDACS receiver and LDACS / DME transmitter $(H)$ with DME antenna gain of $2 \mathrm{~dB}$ and $10 \mathrm{~dB}$ for two different transmission bandwidths, (a) $498 \mathrm{KHz}$, and (b) $732 \mathrm{KHz}$

\section{Throughput Analysis}

In this subsection, the achieved throughput by the proposed LDACS with the existing LDACS is compared and analyzed in the presence of the DME interference. As it can be observed from the PSD results (Fig. 7), that the proposed LDACS has very less interference with the adjacent DME channel, hence it does not require any guard bands for interference reduction.
This helps to achieve higher throughput for the proposed Ref-OFDM based LDACS over the variable distances and altitudes considered above. The achieved throughput values are presented in table III. When the distance and altitude are higher, we get less throughput because of the lower signal power. High throughput performance also supports a higher data rate, which is an essential requirement for future A2GC.

TABLE III. THROUGHPUT ACHIEVED FOR VARIABLE DISTANCE AND ALTITUDE

\begin{tabular}{|l|l|l|l|l|l|}
\hline $\begin{array}{l}\text { Distances } \\
(\mathrm{cm})\end{array}$ & $\begin{array}{l}\text { Throughput } \\
\text { (Mbps) } \\
\text { (existing) }\end{array}$ & $\begin{array}{l}\text { Throughput } \\
\text { (Mbps) } \\
\text { (proposed) }\end{array}$ & $\begin{array}{l}\text { Height } \\
(\mathrm{cm})\end{array}$ & $\begin{array}{l}\text { Throughput } \\
\text { (Mbps) } \\
\text { (existing) }\end{array}$ & $\begin{array}{l}\text { Throughput } \\
\text { (Mbps) } \\
\text { (proposed) }\end{array}$ \\
\hline 5 & 4.2123 & 5.0125 & 10 & 4.9247 & 5.5475 \\
\hline 182 & 3.7215 & 4.8245 & 90 & 1.5894 & 2.3245 \\
\hline 450 & 2.2171 & 4.0548 & 188 & 0.5734 & 0.7581 \\
\hline 945 & 0.7818 & 2.9002 & 493 & 0.1995 & 0.3883 \\
\hline 2450 & 0.0567 & 1.7951 & 766 & 0 & 0 \\
\hline 4205 & 0 & 0 & - & - & - \\
\hline 5940 & 0 & 0 & - & - & - \\
\hline
\end{tabular}

\section{CONCLUSION}

In this paper, we have developed an experimental testbed for the proposed Ref-OFDM based LDACS and compared the PSD, BER and throughput performance with the existing LDACS. The hardware setup includes three NI USRPs for LDACS transmitter, LDACS receiver, and DME transmitter. The signals are transmitted using three VERT 900 antennas. For the performance analysis, different scenarios related to LDACS transmitter/receiver and DME transmitter are considered such as variable distance, variable altitude, LOS, NLOS, and variable DME antenna gain. The proposed Ref-OFDM based LDACS provides nearly $35 \mathrm{~dB}$ less OOB emission and offer better BER/Throughput in all the above-considered scenarios which makes it an alluring substitute to the existing LDACS.

\section{REFERENCES}

[1] M. Schnell, U. Epple, D. Shutin and N. Schneckenburger, "LDACS: future aeronautical communications for air-traffic management," in IEEE Communications Magazine, vol. 52, no. 5, pp. 104-110, May. 2014.

[2] S. Gligorevic, U. Epple, and M. Schnell "The LDACS1 physical layer design," INTECH Open Access Publisher, 2011.

[3] Sajatovic M., Haindl B., Ehammer M., Grupl T., Schnell M., Epple U., and Brandes S., "L-DACS1 System Definition Proposal: Deliverable D2," in Technical Report, Eurocontrol, no. 1.0, Feb 2009.

[4] M. Schnell, N. Franzen and S. Gligorevic, "L-DACS1 laboratory demonstrator development and compatibility measurement set-up," 29th Digital Avionics Systems Conference, Salt Lake City, UT, 2010, pp. 3.E.3-1-3.E.311.

[5] U. Epple and M. Schnell, "Overview of legacy systems in $L$-band and its influence on the future aeronautical communication system LDACS1," in IEEE Aerospace and Electronic Systems Magazine, vol. 29, no. 2, pp. 3137, Feb. 2014.

[6] N. Neji, R. de Lacerda, A. Azoulay, T. Letertre and O. Outtier, "Survey on the Future Aeronautical Communication System and Its Development for Continental Communications," in IEEE Transactions on Vehicular Technology, vol. 62, no. 1, pp. 182-191, Jan. 2013.

[7] N. Schneckenburger, N. Franzen, S. Gligorevic and M. Schnell, "L-band compatibility of LDACS1," IEEE/AIAA 30th Digital Avionics Systems Conference (DASC), pp. 1-11, Seattle, USA, Oct. 2011.

[8] N. Agrawal, S. J. Darak, and F. Bader, "Reconfigurable filtered OFDM waveform for next generation air-to-ground communications," IEEE/AIAA 36th Digital Avionics Systems Conference (DASC), pp. 1-7, St. Petersburg, USA, Sept. 2017.

[9] N. Agrawal, S. J. Darak, and F. Bader, "New Spectrum Efficient Reconfigurable Filtered-OFDM Based $L$-band Digital Aeronautical Communication System,"in IEEE Transactions on Aerospace and Electronic System.

[10] S. Brandes, et al. "Physical layer specification of the $L$-band Digital Aeronautical Communications System (L-DACS1), "IEEE Integrated Communications, Navigation, and Surveillance Conference, pp. 1-12, Arlington, USA, May. 2009. 